\title{
Single-Laser-Pulse-Driven Thermal Limit of the Quasi-Two-Dimensional Magnetic Ordering in $\mathrm{Sr}_{2} \mathrm{IrO}_{4}$
}

\author{
R. Wang $\odot,{ }^{1,2,3}$ J. Sun, ${ }^{1}$ D. Meyers, ${ }^{4,5}$ J. Q. Lin, ${ }^{1,2,3}$ J. Yang, ${ }^{6}$ G. Li,${ }^{1}$ H. Ding, ${ }^{2,3}$ Anthony D. DiChiara, ${ }^{7}$ Y. Cao $\odot,{ }^{8}$ \\ J. Liu, ${ }^{6}$ M. P. M. Dean $\odot,{ }^{4}$ Haidan Wen, ${ }^{7,8}$ and X. Liu $\odot^{1, *}$ \\ ${ }^{1}$ School of Physical Science and Technology, ShanghaiTech University, Shanghai 201210, China \\ ${ }^{2}$ Beijing National Laboratory for Condensed Matter Physics and Institute of Physics, \\ Chinese Academy of Sciences, Beijing 100190, China \\ ${ }^{3}$ University of Chinese Academy of Sciences, Beijing 100049, China \\ ${ }^{4}$ Condensed Matter Physics and Materials Science Department, \\ Brookhaven National Laboratory, Upton, New York 11973, USA \\ ${ }^{5}$ Department of Physics, Oklahoma State University, Stillwater, Oklahoma 74078, USA \\ ${ }^{6}$ Department of Physics and Astronomy, University of Tennessee, Knoxville, Tennessee 37996, USA \\ ${ }^{7}$ Advanced Photon Source, Argonne National Laboratory, Argonne, Illinois 60439, USA \\ ${ }^{8}$ Materials Science Division, Argonne National Laboratory, Argonne, Illinois 60439, USA
}

(Received 12 April 2021; revised 16 August 2021; accepted 3 September 2021; published 2 November 2021)

\begin{abstract}
Upon femtosecond-laser stimulation, generally materials are expected to recover back to their thermalequilibrium conditions, with only a few exceptions reported. Here, we demonstrate that deviation from the thermal-equilibrium pathway can be induced in canonical 3D antiferromagnetically (AFM) ordered $\mathrm{Sr}_{2} \mathrm{IrO}_{4}$ by a single 100 -fs-laser pulse, appearing as losing long-range magnetic correlation along one direction into a glassy condition. We further discover a "critical-threshold ordering" behavior for fluence above approximately $12 \mathrm{~mJ} / \mathrm{cm}^{2}$, which we show corresponds to the smallest thermodynamically stable $c$-axis correlation length needed to maintain long-range quasi-two-dimensional AFM order. We suggest that this behavior arises from the crystalline anisotropy of the magnetic-exchange parameters in $\mathrm{Sr}_{2} \mathrm{IrO}_{4}$, whose strengths are associated with distinctly different timescales. As a result, they play out very differently in the ultrafast recovery processes, compared with the thermal-equilibrium evolution. Thus, our observations are expected to be relevant to a wide range of problems in the nonequilibrium behavior of low-dimensional magnets and other related ordering phenomena.
\end{abstract}

DOI: 10.1103/PhysRevX.11.041023

\section{INTRODUCTION}

Understanding the mechanisms behind laser manipulation of magnetism in materials is indispensable to many problems in both fundamental and applied science [1-5]. Phenomenologically, the classic three-temperature model [5] works quite well in explaining the experimental observations on the evolution of the spins upon external femtosecond-laser stimuli in many systems $[1,5-8]$. In this model, the spin, electron, and lattice baths are coupled through a set of mutual interaction constants which govern the energy-flow rates when the system is stimulated into nonthermal-equilibrium conditions. Eventually, the spin

*liuxr@shanghaitech.edu.cn

Published by the American Physical Society under the terms of the Creative Commons Attribution 4.0 International license. Further distribution of this work must maintain attribution to the author(s) and the published article's title, journal citation, and DOI.
Subject Areas: Condensed Matter Physics

Magnetism, Materials Science sector thermalizes back to equilibrium condition after the externally deposited energy equilibrates among the charge, spin, and lattice reservoirs [5]. Here, we report an exceptional observation of single-laser-pulse-induced anisotropic partial recovery of the magnetic ordering in $\mathrm{Sr}_{2} \mathrm{IrO}_{4}$, leading to permanent reduction of the interplane magnetic correlation. We propose that this unusual recovery behavior could be explained by the noncooperation of different terms of the exchange interactions dictating the spin ordering. When these exchanges differ significantly in strength, they are unfolded along the time axis $[9,10]$ into different timescales in the ultrafast recovery process. Because of this "time-window mismatch," the weaker exchange terms could be quenched, leading to a deviation from the quasiequilibrium relaxation pathway.

$\mathrm{Sr}_{2} \mathrm{IrO}_{4}$ is a layered Mott insulator with each Ir site hosting a $1 / 2$ pseudospin [11]. The highly anisotropic crystalline structure leads to strong magnetic anisotropy [Fig. 1(a)]. The system starts to develop three-dimensional antiferromagnetic (AFM) ordering below $T_{N} \sim 240 \mathrm{~K}$ [12], 
(a)

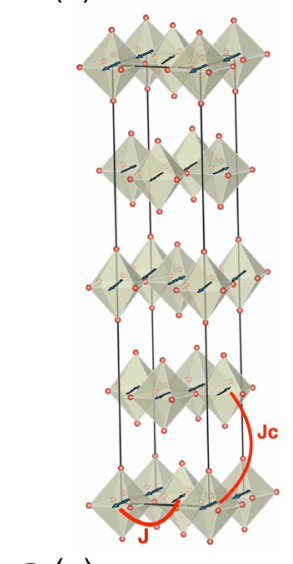

(b)

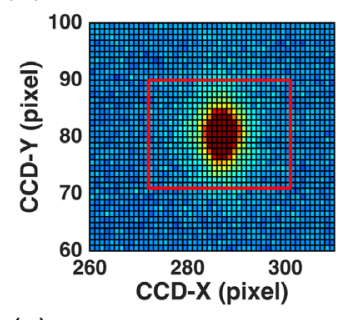

(c)

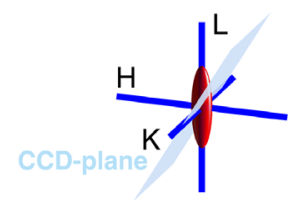

(d)

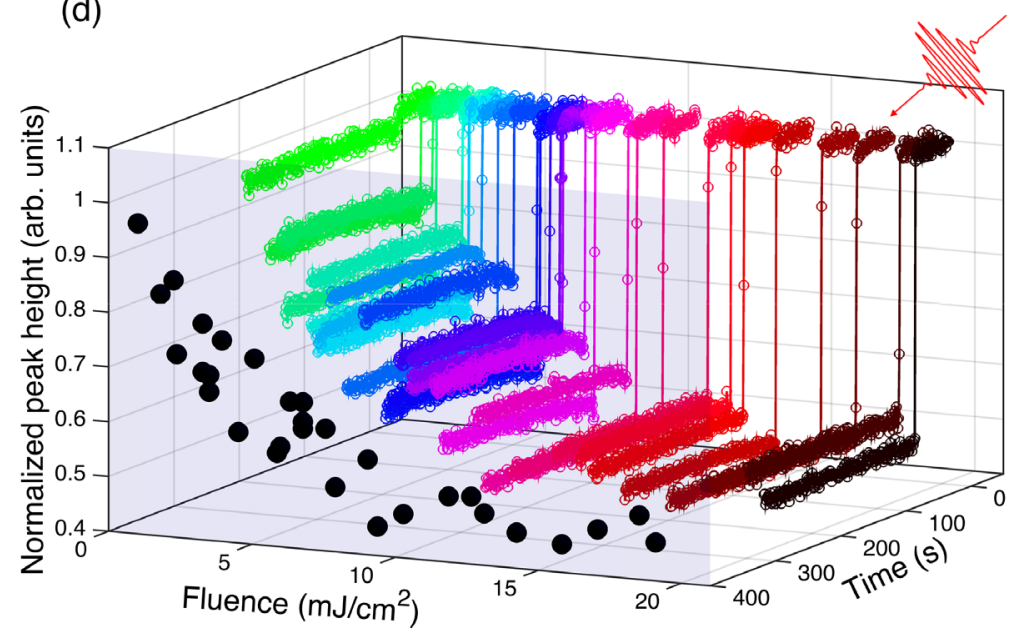

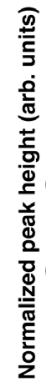
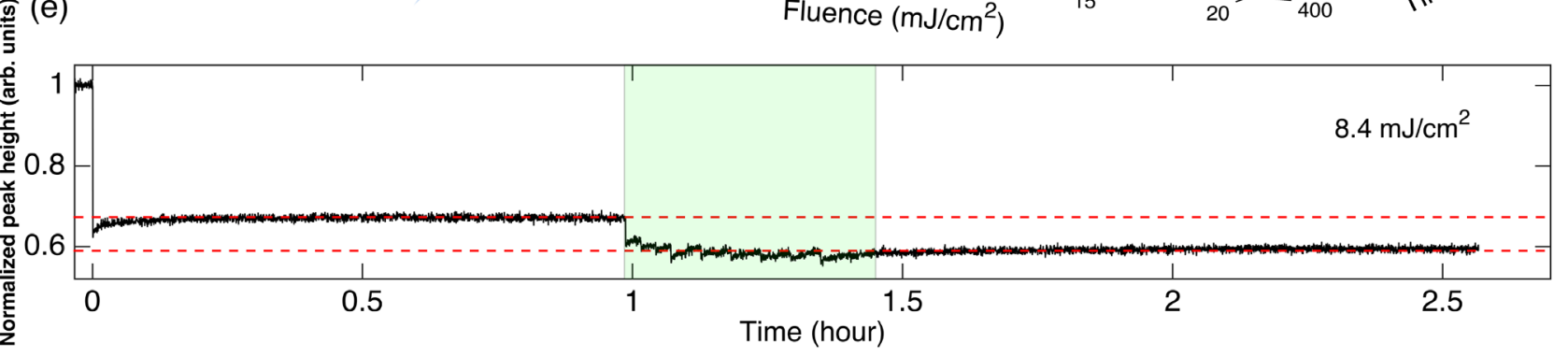

FIG. 1. Single-laser-pulse-induced suppression of the (1 016 ) magnetic reflection. (a) The crystal and magnetic structure of $\mathrm{Sr}_{2} \mathrm{IrO}_{4} . J$ and $J_{c}$ denote the in-plane and interplane exchange couplings, respectively. (b) CCD image of the magnetic peak. (c) Cartoon demonstration of the projection of the CCD plane in the reciprocal space. (d) Response of the magnetic Bragg-peak height to a single laser pulse (arrives at $t=0$ ) as a function of the laser fluence. The black dots are the average of the last $100 \mathrm{~s}$ of each curve. (e) Multipleshot evolution of the magnetic-peak height with moderate laser fluence. Multiple shots arrive in the shaded region, and each creates a small intensity drop until the decrease is fully compensated by an initial recovery.

from the cooperation of a strong intraplane exchange $J$ of approximately $60 \mathrm{meV}$ and a weak interplane exchange $J_{c}$ of approximately $16 \mu \mathrm{eV}[13,14]$. The magnetic ordering in $\mathrm{Sr}_{2} \mathrm{IrO}_{4}$ has been well observed with x-ray resonant magnetic scattering (XRMS) measurements, appearing as Bragg peaks in the magnetic scattering channel when the incident $x$-ray energy was tuned to approximately $11.216 \mathrm{keV}$ at the $\operatorname{Ir}-L_{3}$ resonance edge [15]. This high $\mathrm{x}$-ray resonant energy allows access to a large reciprocal space, thus the flexibility in selecting low $\mathrm{x}$-ray incident angle scattering geometry to best meet the $\mathrm{x}$-ray and laser penetration match requirement $[16,17]$. Our experiment was set up such that our approximately 100-nm epitaxial thin-film sample was stimulated with a 1-eV laser (approximately $100 \mathrm{fs}$ ) pulse by pulse, and the AFM ordering peak was continuously monitored with XRMS at an x-ray incident angle of less than $5^{\circ}$ to track the recovery of the magnetic ordering at a readout frequency of $1 \mathrm{~Hz}$ (see Ref. [18]).

\section{EXPERIMENTAL OBSERVATIONS}

Figure 1(b) shows the (1 0 16) 3D AFM ordering peak in $\mathrm{Sr}_{2} \mathrm{IrO}_{4}$ observed on the pixelated area detector at $80 \mathrm{~K}$ [19], and Fig. 1(c) depicts the detector surface projection in the reciprocal space. In order to catch the true first laser-shot response from pristine thermal-equilibrium condition, each dataset is collected after the sample goes through a fresh thermal cycle by warming above $T_{N}$ and then cooling back again to $80 \mathrm{~K}$ with the laser turned off. Then, the $\mathrm{x}$ ray is turned on to continuously monitor the magnetic Bragg-peak intensity. In between, laser stimuli are controlled to come in pulse by pulse. The arrival time of the first laser pulse is defined as time zero. The evolution of the experimentally observed magnetic-peak height as a function of the laser fluence is shown in Fig. 1(d).

After a single-laser-pulse stimulus to the magnetic ordering prepared from thermal-equilibrium evolution, the system does not recover to the initial state, evidenced by the permanent suppression of the magnetic Bragg-peak height as shown in Fig. 1(d). The degree of the suppression depends on the laser fluence, i.e., the degree of the damage to the initial ordered spin network. More details of the response to the pulsed laser stimulation are shown in Fig. 1(e) with a laser fluence of $8.4 \mathrm{~mJ} / \mathrm{cm}^{2}$. At this fluence, the first laser pulse leads to an approximately $33 \%$ drop in the magnetic Bragg-peak height, and the system settles at this condition without further evolution within an hour of continuous X-ray measurement. A second pulse arriving after one hour causes further suppression to the 

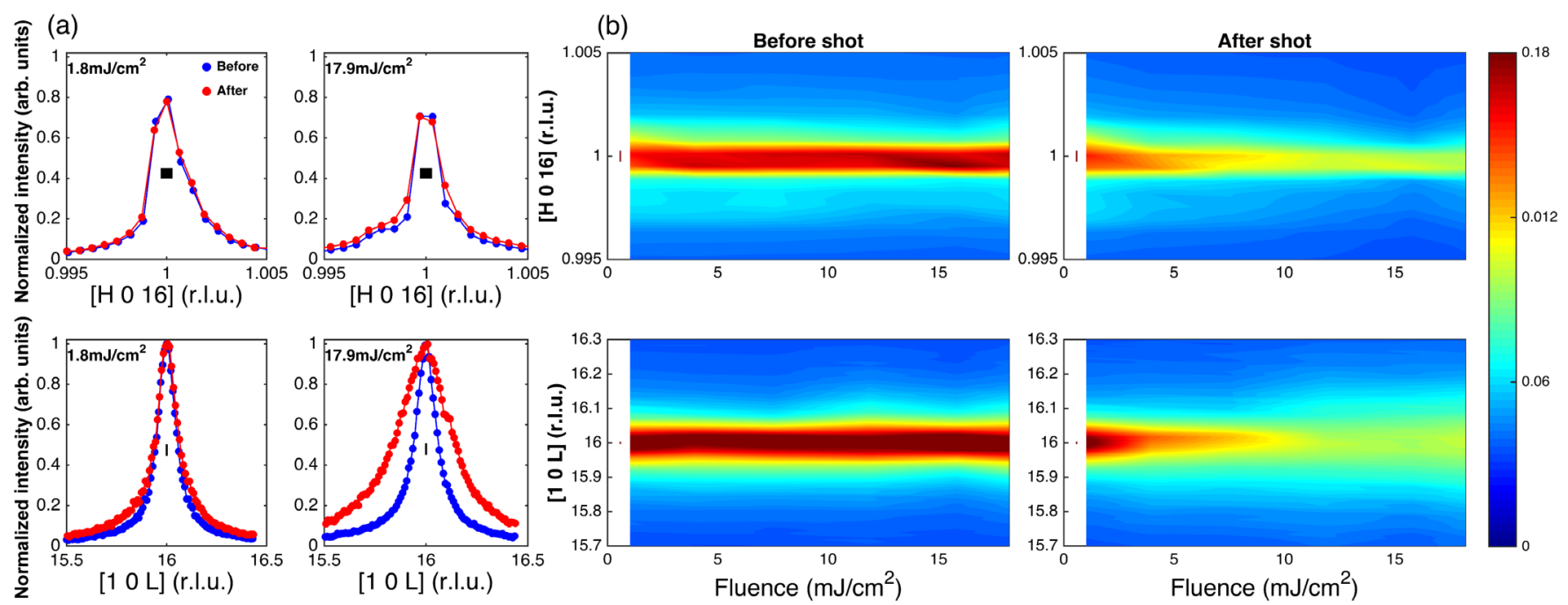

FIG. 2. Evolution of the magnetic ordering upon laser stimulation. (a) Comparison of the $H$ (in-plane) and $L$ (interplane) scans before and after single-pulse-laser pumping. The horizontal bars are our instrumental resolution. Scans are normalized to the peak height determined from $L$ scans to emphasize the variation in the peak widths. (b) Fluence-dependent evolution of the $H$ and $L$ scans.

measured peak height but with a much smaller drop. After 5-6 pulses, the system recovery becomes repeatable, and more stimulation does not cause further permanent peakheight reduction.

These experimental observations clearly demonstrate that the recovery of the magnetic ordering in $\mathrm{Sr}_{2} \mathrm{IrO}_{4}$ depends on the history of laser stimulation, and the response can be classified into two distinct stages. In the first stage of the initial few pulses, the degree of magnetic ordering keeps to be permanently suppressed, and the system does not recover to the condition before pulse arrival. In the second stage, the system does recover but only to a state prepared by multiple initial laser pulses, which, of course, is different from the thermal-equilibrium evolution. The laser-pulse-induced suppression of the magnetic ordering can be erased with a full thermal cycle, and our measurements are fully reproducible (see Ref. [18]), indicating the observed response is not due to sample damage but intrinsic to the spin system in $\mathrm{Sr}_{2} \mathrm{IrO}_{4}$. Phenomenologically, the observed shot-by-shot dependence is similar to the photoinduced metastable insulator-to-metal phase transition in $\mathrm{La}_{2 / 3} \mathrm{Ca}_{1 / 3} \mathrm{MnO}_{3}$ [20]. The laser stimulation drives the system into a nonthermal-equilibrium condition, and the deviation depends on the history of laser stimulation, a non-Markovian-type behavior. Such deviation is obviously beyond the classic three-temperature model [5-8].

To explore the microscopic origin of such evolution, detailed reciprocal-space scans along the in-plane ( $H$ scans) and interplane ( $L$ scans) directions are performed across the magnetic peak before and after the first laser-pulse arrival under various laser fluences, and the results are shown in Fig. 2. Note that the peaks in the $H$ scan, a measure of the in-plane spin ordering, are very sharp and identical before and after the laser pulses for all laser fluences applied. From the width of the peaks in $H$ scans, the in-plane spin-ordering correlation length is estimated to be of a macroscopic scale about $0.9 \mu \mathrm{m}$. Thus, within a layer, the magnetic ordering is always fully restored to the thermalequilibrium condition. The observed permanent suppression in the first stage is due to the incomplete recovery of the spin correlation along the interplane direction, which is evidenced by the broadening of the peaks in $L$ scans. With the spin-correlation length reduced, the scattering intensity is transferred from the center to the tails, leading to a permanent suppression of the peak height as observed. These results suggest that the laser pulse leads to highly anisotropic response between the in-plane and interplane AFM ordering correlations in the recovery process.

To quantify such a response, the $L$ scans of the magnetic Bragg peak measured at thermal-equilibrium condition and after the very first laser-pulse stimulus with varying fluence are analyzed to extract the evolution of the interlayer spinordering texture. It turns out that, for all experimental conditions, the $L$ scans can be well described by a single Lorentzian function as $I\left(q_{z}, \xi_{z}\right) \sim(2 / d)\left(\xi_{z} /\left(1+q_{z}^{2} \xi_{z}^{2}\right)\right)$ [Figs. 3(a) and 3(b) and Ref. [18]], with $d$ the interlayer distance and $q_{z}$ the interlayer direction relative-momentum transfer. The evolution of the extracted correlation length $\xi_{z}$ is shown in Fig. 3(c). As a function of the laser fluence, $\xi_{z}$, as well as the peak height [Fig. 3(d)], generally follows an exponential decay. On the other hand, the total area under the magnetic peak is conserved [Fig. 3(e)]. The significant data fluctuation mostly comes from slight misalignment in the $H$ direction, where the peak width corresponds to $0.01^{\circ}$ in instrument rotational angle. Such conservation indicates that the size and direction of the local ordered magnetic moments are the same as those of the pristine condition 

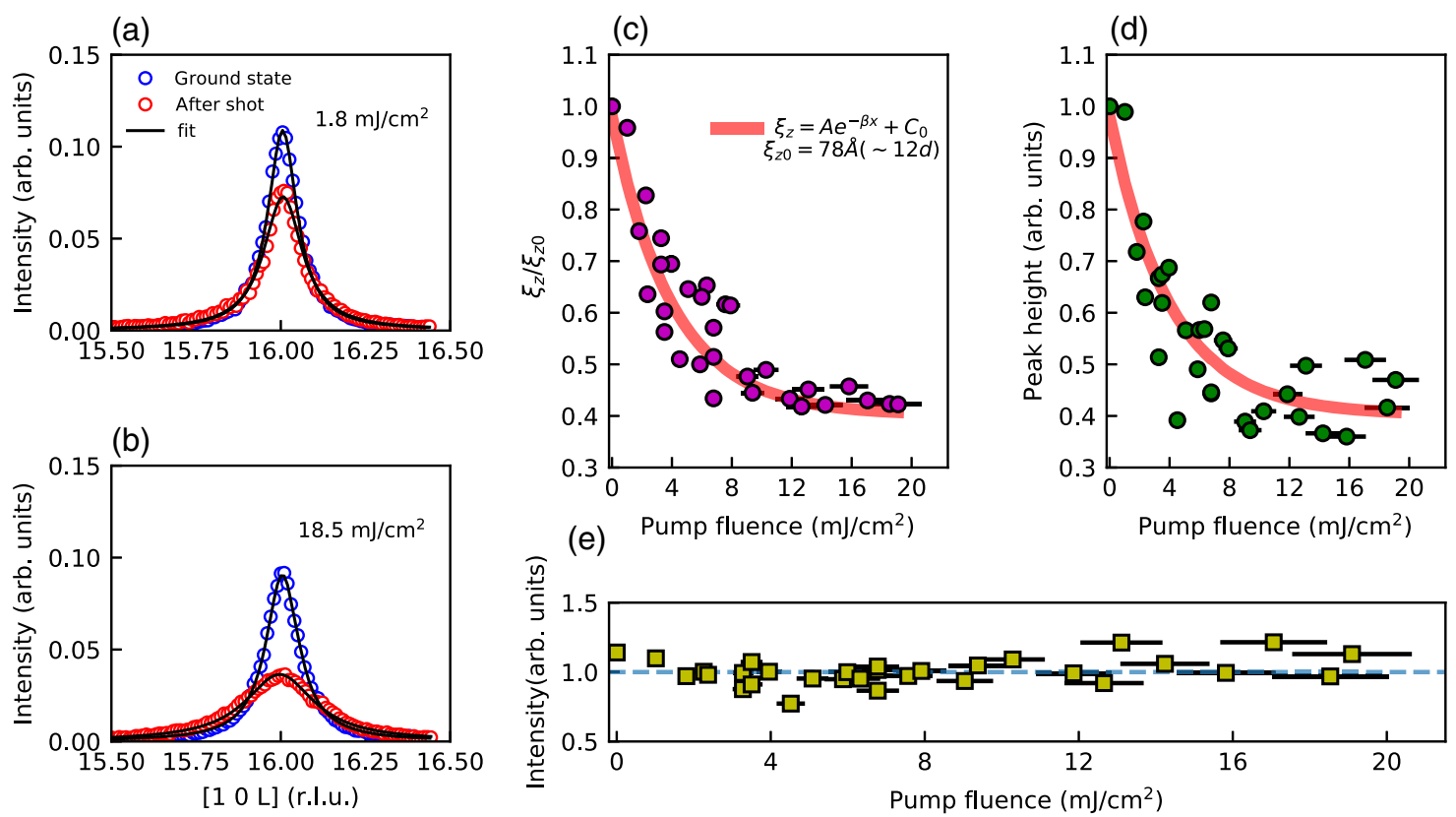

FIG. 3. Evolution of the interplane correlation as a function of the fluence. (a),(b) The fitting results for two representative conditions with weak and strong laser fluence. (c)-(e) The evolution of the interplane correlation length $\xi_{z}$, peak height, and the integrated intensity as a function of the fluence, which are all normalized to the values before laser stimulation. With fluence higher than approximately $12 \mathrm{~mJ} / \mathrm{cm}^{2}, \xi_{z}$ saturates to approximately five-layer thickness. The exponential lines are guides to the eye. The error bar for fluence is estimated to be $10 \%$ (see Supplemental Material [18]).

$[21,22]$. Thus, the incomplete recovery is solely due to the reduction in the interplane correlation. It is interesting to notice that the exponential decay is offset from zero. Both the correlation length and the peak height show a tendency to saturation at higher laser fluence, and the interplane spin correlation cannot be completely destroyed.

\section{DISCUSSION}

Our observations reveal a few interesting aspects of the recovery of the magnetic-ordering texture in this highly magnetically anisotropic layered $\mathrm{Sr}_{2} \mathrm{IrO}_{4}$. First, starting from the thermal-equilibrium condition, each of the first few femtosecond-laser pulses induces permanent suppression to the magnetic ordering, and the degree of the suppression depends on the states prepared by the preceding pulses. Second, the system does eventually stabilize into conditions that the recovery of the magnetism becomes repeatable upon consecutive laser stimulation. This justifies the validity of experiments of stroboscopic mode in probing the spin dynamics $[1,5,17]$. Third, although the degree of the partial recovery keeps degrading with increased laser fluence, it saturates to a robust nonzero value, indicating a minimum interplane partial recovery is intrinsically protected. The observed history and laser-fluence dependence hint at the nature of the magnetic recovery in $\mathrm{Sr}_{2} \mathrm{IrO}_{4}$ upon ultrafast laser stimulation.

The history dependence evidences that the demagnetization has certain local character. In the ultrafast process, the demagnetization does not completely wipe out all traces of magnetic order, and, in the recovery, the spins do not have long enough time to reach a global bath temperature. As a result, there are unperturbed (or less-perturbed) local spin clusters which preserve the memory of the prior state. Thus, the prevailing global energy-dissipation picture in the classic three-temperature model [5-8] is oversimplified.

Notably, in both the initial and the second stages, the inplane spin correlation always recovers to the thermalequilibrium evolution condition of micron size. This differentiates our observation from the conventional fast quenching of a high-temperature state where the correlations along all directions are expected to change [23-25]. The full inplane recovery and the permanent loss of the interplane spin correlation are intimately related to the individual terms in the exchange interaction governing the magnetic dynamics along different directions. Although the 3D magnetic ordering in $\mathrm{Sr}_{2} \mathrm{IrO}_{4}$ is jointly determined by both the intraplane and interplane magnetic couplings $[14,26,27]$, in the ultrafast recovery process, they act differently. The strong in-plane exchange of tens of $\mathrm{meV}$ drives a quick reestablishment of the in-plane correlation within a few picoseconds [17]. We suggest that, in such a short duration, the additional energy introduced by laser pumping into the spin system cannot be efficiently absorbed by the lattice reservoir [28,29]. Instead, the spin sector is still highly excited in the picosecond timescale. Associated with the weak interlayer exchange $J_{c}$ of approximately $16 \mu \mathrm{eV}$, a time window much longer than 
picoseconds is needed to allow them to fully dissipate. Such a process is cut off when the macroscopic intralayer spin correlation is established, since there is an enormous energy barrier to flip a whole layer.

Following the above arguments, we can understand the saturation plateau at high laser fluence as shown in Figs. 3(c) and 3(d). In our earlier report [17], we demonstrate that the laser pulse completely destroys the magnetic order in the initial hundreds of femtoseconds, when the laser fluence is stronger than approximately $12 \mathrm{~mJ} / \mathrm{cm}^{2}$. Thus, beyond such laser fluence, the system completely loses its memory of the history and the recovery follows the same path, regardless of further laser-fluence increasing. This is also consistent with the observed multishot evolution where, at high fluence, the laser pulses after the very first shot drive marginal further suppression to the magnetic Bragg-peak height (see Fig. S9 in Supplemental Material [18]). Without the assistance from the remnant order, reestablishment of the global interplane correlation completely lags behind the intraplane recovery. Thus, the system recovery enters a quasi-two-dimensional regime. For such a condition, Mermin and Wagner [30] prove that spontaneous two-dimensional ferromagnetic or antiferromagnetic long-range order at finite temperature is highly susceptible to spin thermal fluctuations, and the nonzero third-dimension correlation is critical to suppress the thermal fluctuation to realize long-range two-dimensional ordering.

We confirm such an explanation for our observed plateau by calculating the magnetic correlation function in a few layers of square lattice from an effective anisotropic Heisenberg spin-1/2 model, whose dynamics is solved with the equation-of-motion technique and mean-field approximation [31] (see Ref. [18]). With the realistic parameters from experiment and published literature

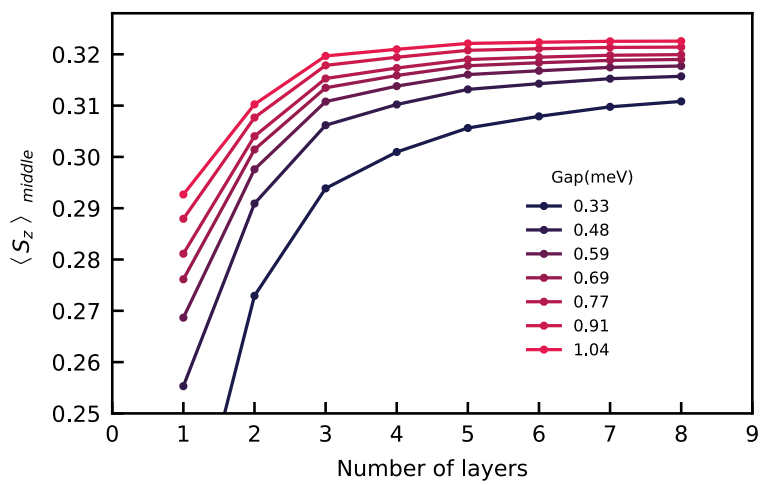

FIG. 4. Theoretical modeling. Calculated magnetic-order parameter at the middle of the slab, $\left\langle S_{z}\right\rangle_{\text {middle }}$, as a function of the layer number from the model Hamiltonian $H=(J / 2) \sum_{l, i j} \vec{S}_{l i} \cdot \vec{S}_{l j}+$ $\Delta \sum_{l, i j} S_{l i}^{z} S_{l j}^{z}+\left(J_{c} / 2\right) \sum_{l l^{\prime}, i j} \vec{S}_{l i} \cdot \vec{S}_{l^{\prime} j}$ at $T=80 \mathrm{~K}$ with exchange terms $J=60 \mathrm{meV}$ and $J_{c}=16.4 \mu \mathrm{eV}$. Different curves correspond to different anisotropic exchange $\Delta$ strengths, and the consequent gap sizes are used as an index.
$[13,14]$, the self-consistent ordered magnetic moment $\left\langle S_{z}\right\rangle$ and the magnetic correlation function $\left\langle S^{-} S^{+}\right\rangle$are obtained as functions of the model slab thickness. As the reported exchange anisotropy for $\mathrm{Sr}_{2} \mathrm{IrO}_{4}$ is quite controversial [14,32-34], the calculated $\left\langle S_{z}\right\rangle_{\text {middle }}$, the ordered magnetic moment at the middle of the slab, is shown in Fig. 4 as a function of the calculated magnon gap size. As expected, both the anisotropic exchange $\Delta$ strengths and the layer thickness are critical for true long-range magnetic ordering. When the gap size approaches approximately $1 \mathrm{meV}$, the magnetic-order parameter $\left\langle S_{z}\right\rangle_{\text {middle }}$ stabilizes around 4-5 layers, which agrees with the interplane correlation length of the saturation plateau we observe in experiment [Figs. 3(c) and 3(d)]. Thus, we realize a spin thermal fluctuation limit in real material with laser-pulse stimulation. Furthermore, this result indicates that indeed the interplane recovery-time window is set by the in-plane spin correlation. The longer interplane correlation established at lower fluence is assisted by memory of the spin network, which is only partially destroyed below the highfluence threshold.

\section{CONCLUSION}

In conclusion, a history and laser-fluence dependence of the partial-recovery process of the 3D AFM ordering in $\mathrm{Sr}_{2} \mathrm{IrO}_{4}$ was observed, which is related to the distinctly different timescales for the interplane and intraplane recoveries in the nonthermal-equilibrium ultrafast process. The noncooperation of the different exchange interactions in the ultrafast process drives the system to deviate from the quasiequilibrium relaxation pathway. Lightinduced deviation from thermal-equilibrium evolution has been rarely observed, and previous reports are associated with the lattice [35] or charge [20,36-38] degrees of freedom. Our results extend the direct observation of deviation from recovery to thermal-equilibrium condition into the spin sector. Furthermore, we suggest that such a time-window mismatch could generally happen in complex systems during ultrafast nonthermal evolution, and our observations could be relevant to a wide range of problems in the nonequilibrium behavior of low-dimensional magnets and related ordering phenomena. For example, the laser-induced "hidden quantum state" in layered $1 T-\mathrm{TaS}_{2}$ could be one special case $[38,39]$.

\section{ACKNOWLEDGMENTS}

We thank Yi Zhu for the assistance of experimental setup. The experimental work by X.L. and R.W. was primarily supported by National Natural Science Foundation of China under Grant No. 11934017. Part of the execution of the experiment and data interpretation by H. W. and Y. C. were supported by the U.S. Department of Energy, Office of Science, Office of Basic Energy Sciences, 
Materials Sciences and Engineering Division. Use of the Advanced Photon Source was supported by the U.S. Department of Energy, Office of Science, Office of Basic Energy Sciences under Contract No. DE-AC0206CH11357. Work at Brookhaven National Laboratory was supported by the U.S. Department of Energy, Office of Science, Office of Basic Energy Sciences, Materials Sciences and Engineering Division under Contract No. DE-SC0012704. J.L. acknowledges support from the National Science Foundation under Grant No. DMR1848269. H. D. acknowledges support from the National Natural Science Foundation of China (No. 11888101). H. D. and X. L. acknowledge support from the Ministry of Science and Technology of China (2016YFA0401000). J. Y. acknowledges funding from the State of Tennessee and Tennessee Higher Education Commission (THEC) through their support of the Center for Materials Processing.

[1] A. Kirilyuk, A. V. Kimel, and T. Rasing, Ultrafast Optical Manipulation of Magnetic Order, Rev. Mod. Phys. 82, 2731 (2010).

[2] A. V. Kimel and M. Li, Writing Magnetic Memory with Ultrashort Light Pulses, Nat. Rev. Mater. 4, 189 (2019).

[3] J. Bigot, M. Vomir, and E. Beaurepaire, Coherent Ultrafast Magnetism Induced by Femtosecond Laser Pulses, Nat. Phys. 5, 515 (2009).

[4] P. Němec, M. Fiebig, T. Kampfrath, and A. V. Kimel, Antiferromagnetic Opto-Spintronics, Nat. Phys. 14, 229 (2018).

[5] E. Beaurepaire, J.-C. Merle, A. Daunois, and J.-Y. Bigot, Ultrafast Spin Dynamics in Ferromagnetic Nickel, Phys. Rev. Lett. 76, 4250 (1996).

[6] Q. Zhang, A. V. Nurmikko, G. X. Miao, G. Xiao, and A. Gupta, Ultrafast Spin-Dynamics in Half-Metallic $\mathrm{CrO}_{2}$ Thin Films, Phys. Rev. B 74, 064414 (2006).

[7] B. Koopmans, G. Malinowski, F. Dalla Longa, D. Steiauf, M. Fähnle, T. Roth, M. Cinchetti, and M. Aeschlimann, Explaining the Paradoxical Diversity of Ultrafast Laserinduced Demagnetization, Nat. Mater. 9, 259 (2010).

[8] J. Kimling, J. Kimling, R. B. Wilson, B. Hebler, M. Albrecht, and D. G. Cahill, Ultrafast Demagnetization of FePt:Cu Thin Films and the Role of Magnetic Heat Capacity, Phys. Rev. B 90, 224408 (2014).

[9] M. Mitrano et al., Ultrafast Time-Resolved X-Ray Scattering Reveals Diffusive Charge Order Dynamics in $\mathrm{La}_{2-x} \mathrm{Ba}_{x} \mathrm{CuO}_{4}$, Sci. Adv. 5, eaax3346 (2019).

[10] C. Bostedt, S. Boutet, D. M. Fritz, Z. Huang, H. J. Lee, H. T. Lemke, A. Robert, W. F. Schlotter, J. J. Turner, and G. J. Williams, Linac Coherent Light Source: The First Five Years, Rev. Mod. Phys. 88, 015007 (2016).

[11] B. J. Kim, H. Jin, S. J. Moon, J.-Y. Kim, B.-G. Park, C. S. Leem, J. Yu, T. W. Noh, C. Kim, S.-J. Oh, J.-H. Park, V. Durairaj, G. Cao, and E. Rotenberg, Novel $\mathrm{J}_{\mathrm{eff}}=1 / 2$ Mott State Induced by Relativistic Spin-Orbit Coupling in $\mathrm{Sr}_{2} \mathrm{IrO}_{4}$, Phys. Rev. Lett. 101, 076402 (2008).

[12] M. K. Crawford, M. A. Subramanian, R. L. Harlow, J. A. Fernandez-Baca, Z. R. Wang, and D. C. Johnston, Struc- tural and Magnetic Studies of $\mathrm{Sr}_{2} \mathrm{IrO}_{4}$, Phys. Rev. B 49, 9198 (1994).

[13] J. Kim, D. Casa, M. H. Upton, T. Gog, Y.-J. Kim, J. F. Mitchell, M. van Veenendaal, M. Daghofer, J. van den Brink, G. Khaliullin, and B. J. Kim, Magnetic Excitation Spectra of $\mathrm{Sr}_{2} \mathrm{IrO}_{4}$ Probed by Resonant Inelastic X-Ray Scattering: Establishing Links to Cuprate Superconductors, Phys. Rev. Lett. 108, 177003 (2012).

[14] J. Porras, J. Bertinshaw, H. Liu, G. Khaliullin, N. H. Sung, J.-W. Kim, S. Francoual, P. Steffens, G. Deng, M. Moretti Sala, A. Efimenko, A. Said, D. Casa, X. Huang, T. Gog, J. Kim, B. Keimer, and B. J. Kim, Pseudospin-Lattice Coupling in the Spin-Orbit Mott Insulator $\mathrm{Sr}_{2} \mathrm{IrO}_{4}$, Phys. Rev. B 99, 085125 (2019).

[15] B. J. Kim, H. Ohsumi, T. Komesu, S. Sakai, T. Morita, H. Takagi, and T.-H. Arima, Phase-Sensitive Observation of a Spin-Orbital Mott State in $\mathrm{Sr}_{2} \mathrm{IrO}_{4}$, Science 323, 1329 (2009).

[16] B. Guzelturk, T. Winkler, T. W. J. Van de Goor, M. D. Smith, S. A. Bourelle, S. Feldmann, M. Trigo, S. W. Teitelbaum, H. G. Steinrück, G. A. de la Pena, R. Alonso-Mori, D. Zhu, T. Sato, H. I. Karunadasa, M. F. Toney, F. Deschler, and A. M. Lindenberg, Visualization of Dynamic Polaronic Strain Fields in Hybrid Lead Halide Perovskites, Nat. Mater. 20, 618 (2021).

[17] M. Dean, Y. Cao, X. Liu, S. Wall, D. Zhu, R. Mankowsky, V. Thampy, X. M. Chen, J. G. Vale, D. Casa et al., Ultrafast Energy- and Momentum-Resolved Dynamics of Magnetic Correlations in the Photo-Doped Mott Insulator $\mathrm{Sr}_{2} \mathrm{IrO}_{4}$, Nat. Mater. 15, 601 (2016).

[18] See Supplemental Material at http://link.aps.org/ supplemental/10.1103/PhysRevX.11.041023 for details on experiments, data analysis, and model calculation.

[19] A. Lupascu, J. P. Clancy, H. Gretarsson, Z. Nie, J. Nichols, J. Terzic, G. Cao, S. S. A. Seo, Z. Islam, M. H. Upton, J. Kim, D. Casa, T. Gog, A. H. Said, V. M. Katukuri, H. Stoll, L. Hozoi, J. van den Brink, and Y.-J. Kim, Tuning Magnetic Coupling in $\mathrm{Sr}_{2} \mathrm{IrO}_{4}$ Thin Films with Epitaxial Strain, Phys. Rev. Lett. 112, 147201 (2014).

[20] J. Zhang, X. Tan, M. Liu, S. W. Teitelbaum, K. W. Post, F. Jin, K. A. Nelson, D. Basov, W. Wu, and R. D. Averitt, Cooperative Photoinduced Metastable Phase Control in Strained Manganite Films, Nat. Mater. 15, 956 (2016).

[21] M. F. Collins, Magnetic Critical Scattering (Oxford University, New York, 1989).

[22] A. Fluerasu, M. Sutton, and E. M. Dufresne, X-Ray Intensity Fluctuation Spectroscopy Studies on Phase-Ordering Systems, Phys. Rev. Lett. 94, 055501 (2005).

[23] A. J. Bray, Theory of Phase-Ordering Kinetics, Adv. Phys. 43, 357 (1994).

[24] G. F. Mazenko, Theory of Unstable Growth, Phys. Rev. B 42, 4487 (1990).

[25] P. J. Shah and O. G. Mouritsen, Dynamics of Ordering Processes in Annealed Dilute Systems: Island Formation, Vacancies at Domain Boundaries, and Compactification, Phys. Rev. B 41, 7003 (1990).

[26] M. A. Kastner, R. J. Birgeneau, G. Shirane, and Y. Endoh, Magnetic, Transport, and Optical Properties of Monolayer Copper Oxides, Rev. Mod. Phys. 70, 897 (1998). 
[27] S. Fujiyama, H. Ohsumi, T. Komesu, J. Matsuno, B. J. Kim, M. Takata, T. Arima, and H. Takagi, Two-Dimensional Heisenberg Behavior of $\mathrm{J}_{\mathrm{eff}}=1 / 2$ Isospins in the Paramagnetic State of the Spin-Orbital Mott Insulator $\mathrm{Sr}_{2} \mathrm{IrO}_{4}$, Phys. Rev. Lett. 108, 247212 (2012).

[28] J. Li, R. Wang, H. Guo, Y. Zhu, Y. Cao, J. Liu, H. Ding, H. Wen, and X. Liu, Recovery of Photoexcited Magnetic Ordering in $\mathrm{Sr}_{2} \mathrm{IrO}_{4}$, J. Phys. Condens. Matter 31, 25580 (2019).

[29] Y. Li, R. D. Schaller, M. Zhu, D. A. Walko, J. Kim, X. Ke, L. Miao, and Z. Q. Mao, Strong Lattice Correlation of NonEquilibrium Quasiparticles in a Pseudospin-1/2 Mott Insulator $\mathrm{Sr}_{2} \mathrm{IrO}_{4}$, Sci. Rep. 6, 19302 (2016).

[30] N. Mermin and H. Wagner, Absence of Ferromagnetism or Antiferromagnetism in One- or Two-Dimensional Isotropic Heisenberg Models, Phys. Rev. Lett. 17, 1133 (1966).

[31] H. Diep, Quantum Effects in Heisenberg Antiferromagnetic Thin Films, Phys. Rev. B 43, 8509 (1991).

[32] Y. Gim, A. Sethi, Q. Zhao, J. F. Mitchell, G. Cao, and S. L. Cooper, Isotropic and Anisotropic Regimes of the FieldDependent Spin Dynamics in $\mathrm{Sr}_{2} \mathrm{IrO}_{4}$ : Raman Scattering Studies, Phys. Rev. B 93, 024405 (2016).

[33] S. Calder, D. M. Pajerowski, M. B. Stone, and A. F. May, Spin-Gap and Two-Dimensional Magnetic Excitations in $\mathrm{Sr}_{2} \mathrm{IrO}_{4}$, Phys. Rev. B 98, 220402(R) (2018).
[34] D. Pincini, J. G. Vale, C. Donnerer, A. de la Torre, E. C. Hunter, R. Perry, M. M. Sala, F. Baumberger, and D. F. McMorrow, Anisotropic Exchange and Spin-Wave Damping in Pure and Electron-Doped $\mathrm{Sr}_{2} \mathrm{IrO}_{4}$, Phys. Rev. B 96, 075162 (2017).

[35] V. A. Stoica, N. Laanait, C. Dai et al., Optical Creation of a Supercrystal with Three-Dimensional Nanoscale Periodicity, Nat. Mater. 18, 377 (2019).

[36] V. Kiryukhin, D. Casa, J. Hill, B. Keimer, A. Vigliante, Y. Tomioka, and Y. Tokura, An X-Ray-Induced InsulatorMetal Transition in a Magnetoresistive Manganite, Nature (London) 386, 813 (1997).

[37] S.-Y. Xu, Q. Ma, Y. Gao, A. Kogar, A. Zong, A. M. M. Valdivia, T. H. Dinh, S.-M. Huang, B. Singh, C.-H. Hsu et al., Spontaneous Gyrotropic Electronic Order in a Transition-Metal Dichalcogenide, Nature (London) 578, 545 (2020).

[38] L. Stojchevska, I. Vaskivskyi, T. Mertelj, P. Kusar, D. Svetin, S. Brazovskii, and D. Mihailovic, Ultrafast Switching to a Stable Hidden Quantum State in an Electronic Crystal, Science 344, 177 (2014).

[39] S.-H. Lee, J. S. Goh, and D. Cho, Origin of the Insulating Phase and First-Order Metal-Insulator Transition in 1T-TaS 2 , Phys. Rev. Lett. 122, 106404 (2019). 